\title{
Can Using an Intensive Management Program Improve Primary Care Staff Experiences With Caring for High-Risk Patients?
}

\author{
Lisa S. Meredith, PhD; Gulrez Azhar, MD, PhD; Evelyn T. Chang, MD, MSHS; Adeyemi Okunogbe, PhD; \\ Alissa Simon, MA; Bing Han, PhD; and Lisa V. Rubenstein, MD, MSPH
}

\begin{abstract}
Background: Complex, high-risk patients present challenges for primary care staff. Intensive outpatient management teams aim to serve as a resource for usual primary care to improve care for high-risk patients without adding burden to the primary care staff. Whether such assistance can influence the primary care staff experiences is unknown. The objective of this study was to examine improvement in job satisfaction and intent to stay for primary care staff at the US Department of Veterans Affairs (VA) who sought assistance from an intensive management program.
\end{abstract}

Methods: Longitudinal analysis of a staff cohort that completed 2 cross-sectional surveys 18 months apart, controlling for outcomes at time 1. Participants included 144 primary care providers at 5 geographically diverse VA health care systems who completed both surveys. Measured outcomes included job satisfaction and intent to stay within primary care at the VA (measured at time 2). Predictors included likelihood of using intensive management teams (measured at time 1). Covariates included outcomes and professional/practice characteristics (measured at time 1).

Results: The response rate for primary care staff that completed both surveys was $21 \%$. Staff who indicated at time 1 that they were more likely to use intensive management teams for high-risk patients reported significantly higher satisfaction and intention to stay at VA primary care at time 2 (both $P<.05$ ).

Conclusions: A VA primary care workforce might benefit from assistance from intensive management teams for high-risk patients. Additional work is needed to understand the mechanisms by which primary care staff benefit and how to optimize them.
Author affiliations can be found at the end of the article.

Correspondence: Lisa Meredith

(lisa_meredith@rand.org)

Fed Pract. 2021;38(2):68-73. doi:10.12788/fp.0090
$\mathrm{P}$ atients with complex medical and psychosocial needs are at the highest risk for fragmented care and adverse health outcomes. ${ }^{1,2}$ Although these highrisk patients make up only about $5 \%$ of the US patient population, they can account for as much as half of total health care costs. ${ }^{1}$ High-risk patients are complicated to treat because most have multiple chronic medical conditions, and many have a wide variety of psychological and social needs. Thus, physician, physician assistant, and nurse practitioner primary care providers (PCPs), and nurses (registered nurses, licensed vocational nurses, and licensed practical nurses) must address the complexity of the human condition in conjunction with health problems. ${ }^{2}$

\section{BACKGROUND}

Caring for high-risk patients within a tight clinic schedule geared to the provision of comprehensive care to large panels of less complex patients can be a source of stress for PCPs and nurses. ${ }^{3-5}$ These conditions may lead to reduced well-being among primary care team members and to potential turnover. ${ }^{6}$ Furthermore, primary care staff may feel uncomfortable or lack the ability to address nonmedical concerns because of "person-specific factors that interfere with the delivery of usual care and decision making for whatever condition the patient has." 7,8 Having additional support for complex patients, such as intensive outpatient management teams, may be protective both by reducing health care provider (HCP) stress and improving patient outcomes. ${ }^{3,4}$

Caring for high-risk patients is challenging. ${ }^{9-11}$ High-risk patient care may require additional, often unpaid, work hours and may be discouraging because these patients can be difficult to engage in care. ${ }^{7,12}$ Furthermore, high-risk patient care is challenging for primary care teams, since these complex patients may fall through the cracks and experience potentially preventable hospitalization or even death. Avoiding these negative consequences typically requires substantial time for the primary care team to engage and counsel the patient, family, and caregiver, through more frequent visits and additional communication. Furthermore, the primary care team typically must coordinate with other HCPs and resources-as many as 16 in a single year and as much as 12 for a single patient over an 80-day period. ${ }^{13,14}$ Not surprisingly, primary care teams identify help with care coordination as a critical need that may be addressed with intensive management support. 
Primary care at the US Department of Veterans Affairs (VA) Veterans Health Administration (VHA) provides care for a large proportion of high-risk patients. ${ }^{15}$ Accordingly, VHA provides a variety of intensive management options for equipping primary care teams with expanded resources for caring for high-risk patients, including those offered in a few sites by a pilot intensive management program. ${ }^{16}$ As part of the pilot's evaluation, we studied the work experiences of PCPs and nurses, some of whom had experienced the pilot program and some of whom only had access to typical VHA intensive management resources, such as telehealth and specialty medical homes (referred to in the VA as patient aligned care teams, or PACT), eg, for women patients, for patients who are homeless, or for older adults. ${ }^{17}$ Surveys assessed whether HCPs who indicated they were likely to seek help from PACT intensive management (PIM) teams to care for high-risk patients had higher job satisfaction and intention to stay at VHA compared with those who were not likely to seek help.

While substantial research on high-risk patients' intensive management needs has focused on patient-level outcomes of interventions for meeting those needs, little research has examined links between primary care team access to intensive management resources and experiences, such as job satisfaction and job retention. ${ }^{18}$ In the work presented here, our objectives were to (1) assess the likelihood that a PCP or nurse intent to manage high-risk patients by seeking care coordination help from or transferring care to an intensive management team; and (2) evaluate the relationship between PCP or nurse intentions regarding using intensive management help for high-risk patients and their job satisfaction and likelihood of leaving VA primary care. We hypothesized that the accessibility of intensive management resources and PCP and nurse receptivity to accessing those resources may affect job-related experiences.

\section{METHODS}

This study was conducted as part of the evaluation of a VA pilot project to provide
TABLE 1 Characteristics of Primary Care Staff in the Longitudinal Cohort (Completed Both Surveys) Compared With the Staff in Other Samples

Surveys, No. (\%)

\begin{tabular}{lcccc} 
Characteristics & Both $(\mathbf{n = 1 4 4 )}$ & Single $(\mathbf{n}=\mathbf{4 4 2})$ & Neither $(\mathbf{n}=\mathbf{6 4 5})$ & $\boldsymbol{P ~ V a l u e ~}^{\mathbf{a}}$ \\
\hline $\begin{array}{l}\text { Staff type } \\
\text { Physician }\end{array}$ & $36(25.0)$ & $117(26.6)$ & $220(34.1)$ & $<.05$ \\
$\begin{array}{l}\text { Physician assistant or } \\
\text { nurse practitioner }\end{array}$ & $15(10.4)$ & $45(10.2)$ & $55(8.5)$ & \\
$\begin{array}{l}\text { Nurse, registered } \\
\text { Nurse, licensed practical }\end{array}$ & $57(39.6)$ & $155(35.2)$ & $186(28.8)$ & \\
Medical center & $36(25.0)$ & $123(28.0)$ & $184(28.5)$ & \\
A & & & & $<.001$ \\
B & $36(25.0)$ & $95(21.5)$ & $176(27.3)$ & \\
C & $32(22.2)$ & $133(30.1)$ & $174(27.0)$ & \\
D & $27(18.8)$ & $81(18.3)$ & $113(17.5)$ & \\
E & $13(9.0)$ & $74(16.7)$ & $91(14.1)$ & \\
\hline PACT type & $36(25.0)$ & $59(13.4)$ & $91(14.1)$ & \\
Primary care & & & & \\
Speciality care & $113(78.5)$ & $326(73.8)$ & $494(76.6)$ & \\
\hline M facility & $31(21.5)$ & $116(26.2)$ & $151(23.4)$ & \\
\hline Women & $47(32.6)$ & $166(37.6)$ & $175(27.1)$ & $<.01$ \\
\hline
\end{tabular}

Abbreviations: PACT, patient aligned care team; IM, intensive management.

Calculated for difference between staff who responded to both surveys compared with those who responded to 1 or neither survey.

Vomen's health, geriatrics, or infectious disease. general primary care teams with intensive management support from interdisciplinary teams for high-risk patients in 5 VHA systems in 5 states (Ohio, Georgia, North Carolina, Wisconsin, and California). ${ }^{6}$ We sampled primary care staff at 39 primary care clinics within those systems, all of whom had access to VA intensive management resources. These included telehealth, health coaches, integrated mental health providers, and specialty PACTs for specific populations (eg, those who are women, elderly, homeless, HIV-positive, or who have serious mental illness). Of the 39 primary care clinics that participated in the survey, 8 also participated in the pilot program offering an intensive management team to support general primary care in their care of high-risk patients.

Data are from PCPs and nurses who completed 2 cross-sectional surveys (online or hard copy). We invited 1,000 PCPs and nurses to complete the first survey (fielded December 2014 to May 2015) and 863 to complete the second survey (fielded October 2016 to January 2017). A total of 
TABLE 2 Overall Job Satisfaction and Intent to Continue Working at the VA for the Next 2 Years at Time $2(n=144)$

\begin{tabular}{|c|c|c|c|c|c|}
\hline \multirow[b]{2}{*}{ Variables } & \multirow[b]{2}{*}{ Results } & \multicolumn{2}{|c|}{ Job Satisfaction } & \multicolumn{2}{|c|}{ Intent to Stay at VA } \\
\hline & & Coefficient & SE & Coefficient & SE \\
\hline Likely to use intensive management team for HRPs, No. (\%) & $103(71.53)$ & $0.63^{a}$ & 0.21 & $0.41^{\mathrm{b}}$ & 0.18 \\
\hline Works at facility with intensive management teams, No. (\%) & $47(32.64)$ & 0.33 & 0.20 & 0.18 & 0.24 \\
\hline Women (vs men), No. (\%) & $118(81.94)$ & -0.40 & 0.33 & -0.32 & 0.17 \\
\hline PCPs (vs nurses), No. (\%) & $51(35.42)$ & -0.28 & 0.21 & 0.24 & 0.15 \\
\hline Years practicing at current VA clinic, mean (SD) & $7.44(6.15)$ & -0.01 & 0.01 & $-0.02^{a}$ & 0.01 \\
\hline Fully staffed team (vs partially), No. (\%) & $101(70.14)$ & 0.12 & 0.16 & -0.16 & 0.15 \\
\hline $11-20 \%$ HRPs on panel (vs 0-10\%), No. (\%) & $43(29.86)$ & 0.34 & 0.21 & 0.16 & 0.21 \\
\hline$>20 \%$ HRPs on panel (vs 0-10\%), No. (\%) & $64(44.44)$ & -.21 & 0.18 & -0.19 & 0.21 \\
\hline Outcome at Time 1 , mean (SD) ${ }^{d}$ & $\begin{array}{l}3.71(1.07) \\
4.08(0.98)\end{array}$ & $0.40^{c}$ & 0.09 & $0.28^{a}$ & 0.09 \\
\hline$R^{2}$ & -- & 0.28 & -- & 0.22 & -- \\
\hline
\end{tabular}

Abbreviations: HRP, high-risk patient; PCP, primary care providers; SE, standard error; VA, US Department of Veterans Affairs.

${ }^{a} P<.01$.

${ }^{\mathrm{b}} P<.05$.

${ }^{c} P<.001$.

dJob satisfaction or intent to stay.

436 completed the first survey for a response rate of $44 \%$, and 313 completed the second survey for a response rate of $36 \%$. We constructed a longitudinal cohort of 144 PCPs and nurses who completed both surveys and had data at 2 timepoints. This longitudinal cohort represents 33\% of the 442 unique respondents who completed either the first or second survey. Overlap across surveys was low because of high staff turnover between survey waves.

\section{Measures}

Outcomes. We examined 2 single-item outcome measures to assess job satisfaction and retention (ie, intent to stay in primary care at the VA) measured in both surveys. These items were worded "Overall, I am satisfied with my job." and "I intend to continue working in primary care at the VA for the next two years." Both items were rated on a 5-point Likert scale.

Independent Variable. We assessed proclivity to seek assistance in caring for highrisk patients based on PCPs or nurses indicating that they are likely to either "manage these patients with ongoing care coordination assistance from an intensive management team" and/or "transfer these patients from primary care to another intensive management team or program specializing in high-risk patients." These 2 items were rated on a 5-point Likert scale; we dichotomized the scale with likely or very likely indicating high proclivity (likelihood) for ease of interpretation of the combined items.

Covariates. We also controlled for indicators of staff demographic and practice characteristics in multivariate analyses. These included gender, staff type (PCP vs nurse), years practicing at a VA clinic, team staffing level (full vs partial), proportion of the panel consisting of high-risk patients (using binary indicators: 11 to $20 \%$ or $>20 \%$ compared with 0 to $10 \%$ as the reference group), and whether or not the site participated in the pilot program offering an intensive management team to support primary care for high-risk patients to distinguish the 8 pilot sites from nonpilot sites. 


\section{Statistical Analysis}

We used ordinary least squares regression analysis to examine associations between the independent variable measured at time 1 and outcomes measured at time 2, controlling for time 1 outcomes among staff who completed both surveys (eg, the longitudinal cohort). We adjusted for time 1 covariates and clustering of staff within clinics, assuming a random effect with robust standard errors, and conducted multiple imputations for itemlevel missing data. Poststratification weights were used to adjust for survey nonresponse by staff type, gender, facilities participating in the innovations, and type of specialty PACT. We calculated weights based on the sampling frame of all PCPs and nurses for each survey.

\section{RESULTS}

Table 1 shows the proportion of primary care staff responding to the surveys. For the longitudinal cohort, the response by staff type was similar to the sample of staff that responded only to a single survey, but the sample that did not respond to either survey included more physicians. There was also some variation by medical center. For example, a smaller proportion of the cohort was from site D and more was from site E compared with the other samples. The proportion of primary care staff in facilities that participated in the intensive management pilot was higher than the proportion in other facilities. More women $(81.9 \%)$ were in the longitudinal cohort compared with $77.4 \%$ in the single-survey sample and $69.2 \%$ in the sample that responded to neither survey.

Both surveys were completed by 144 respondents while 442 completed 1 survey and 645 did not respond to either survey. The cohort was predominantly nurses (64.6\%); Of the PCPs, $25 \%$ were physicians. Most staff were women $(81.9 \%)$ and aged $>45$ years $(72.2 \%)$. Staff had practiced at their current VA clinics for a mean of 7.4 years, and most reported being on a fully-staffed primary care team $(70 \%)$.

\section{Multivariate Analyses}

In the multivariable regression analyses, we found that the primary care staff, which reported being more likely to use intensive management teams to help care for highrisk patients at time 1 , reported signifi-
FIGURE Impact of Intensive Management Team Use on Job Satisfaction and Intent to Stay at the VA

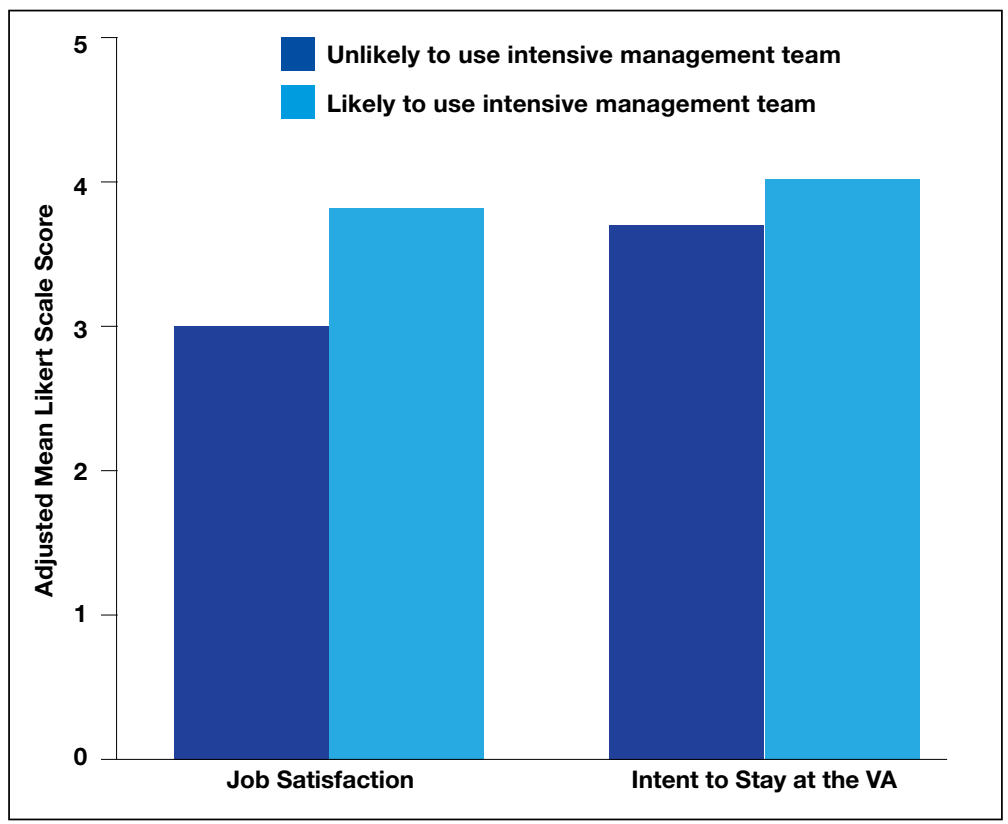

Abbreviation: VA, US Department of Veterans Affairs

cantly higher satisfaction ( 0.63 points higher on a 5-point scale) and intention to stay $(0.41$ points higher) at VA primary care (both $P<.05$ ) at time 2,18 months later (Table 2). These effect sizes are equivalent to nearly two-thirds and half of a standard deviation, respectively. Among our control variables, years practicing in the VA was significantly associated with a lower likelihood of intent to stay at the VA. Models account for $28 \%$ of the variation in satisfaction and $22 \%$ of the variation in retention. The Figure shows the adjusted means based on parameters from the regression models for job satisfaction and intent to stay at the VA as well as likelihood of using an intensive management team for high-risk patients. Job satisfaction is nearly 1 point higher among those who report being likely to draw on support from an intensive management team to care for high-risk patients compared with those who reported that they were unlikely to use such a team. The pattern for intent to stay at the VA, while less pronounced, is similar to that for satisfaction.

\section{DISCUSSION}

Our findings are consistent with our hypothesis that augmenting primary care with highrisk patient intensive management assistance 
would improve primary care staff job satisfaction and retention. Findings also mirror recent qualitative studies, which have found that systemic approaches to augment primary care of high-risk patients are likely needed to maintain well-being. ${ }^{719}$ We found a positive relationship between the likelihood of using intensive management teams to help care for their high-risk patients and reported job satisfaction and intent to continue to work within VA primary care 18 months later. To our knowledge, this study is the first to examine the potential impact on PCPs and nurses of using intensive management teams to help care for high-risk patients.

Our study suggests that this approach has the potential to alleviate PCP and nurse stress by incorporating intensive management teams as an extension of the medical home. Even high-functioning medical homes may find it challenging to meet the needs of their high-risk patients. ${ }^{3,7,8}$ Time constraints and a structured clinic schedule may limit the ability of medical homes to balance the needs of the general panel vs the individual needs of high-risk patients who might benefit from intensive services. Limited knowledge and lack of training to address the broad array of problems faced by high-risk patients also makes care challenging. ${ }^{2}$

Intensive management services often include interdisciplinary and comprehensive assessments, care coordination, health care system navigation, and linkages to social and home care services. ${ }^{20}$ Medical homes may benefit from these services, especially resources to support care coordination and communication with specialists and social services in large medical neighborhoods. ${ }^{21}$ For example, including a social worker on the intensive patient care team can help primary care staff by focusing specialized resources on nonmedical issues, such as chronic homelessness, substance use disorders, food insecurity, access to transportation, and poverty. ${ }^{18}$

\section{Limitations}

This study is subject to some limitations, including those typical of surveys, such as reliance on self-reported data. The longitudinal sample we studied had response rates that varied by site, participation in the pilot program, and gender relative to those who did not respond to both surveys; selection bias is possible. While we use a longitudinal cohort, we cannot attribute causality; it is possible that more satisfied staff are more likely to use intensive management teams rather than the use of intensive management teams contributing to higher satisfaction. Although each study site includes at least 1 type of intensive management resource, we cannot ascertain which intensive management resource primary care staff accessed, if any. While our sample size for the longitudinal cohort responders was limited, focusing on our longitudinal cohort provides more valid and reliable estimates than does using 2 crosssectional samples with all responders. In addition, our models do not completely explain variation in the outcomes $\left(R^{2}=0.28\right.$ and 0.22), although we included major explanatory factors, such as team staffing and professional type; other unmeasured factors may explain our outcomes. Finally, our provider sample may not generalize to HCPs in non-VA settings.

\section{CONCLUSIONS}

Our study expands on the limited data regarding the primary care staff experience of caring for high-risk patients and the potential impact of using interdisciplinary assistance to help care for this population. A strength of this study is the longitudinal cohort design that allowed us to understand staff receptivity to having access to intensive management resources to help care for high-risk patients over time among the same group of primary care staff. Given that an economic analysis has determined that the addition of the pilot intensive management program has been cost neutral to the VA, the possibility of its benefit, as suggested by our study findings, would support further implementation and evaluation of intensive management teams as a resource for PCPs caring for high-risk patients. ${ }^{22}$

Understanding the mechanisms by which primary care staff benefit most from highrisk patient assistance, and how to optimize communication and coordination between primary care staff and intensive management teams for high-risk patients might further increase primary care satisfaction and retention. 


\section{Author affiliations}

Lisa Meredith is a Senior Behavioral Scientist at the RAND Corporation, Professor, Pardee RAND Graduate School, and Research Scientist at the VA Center for the Study of Healthcare Innovation, Implementation \& Policy in Santa Monica, California. Gulrez Azhar is a Senior Fellow, Futures Health Scenarios at the Institute for Health Metrics and Evaluation, University of Washington and an Adjunct Policy Researcher at RAND. Evelyn Chang is a Primary Care Physician and Health Services Researcher at VA Greater Los Angeles Health System (VAGLAHS) and an Assistant Clinical Professor in Health Sciences at University of California in Los Angeles (UCLA). Adeyemi Okunogbe is a Health Systems Specialist at RTI International, Washington, DC. Alissa Simon is a Health Science Specialist at the VAGLAHS. Bing Han is a Senior Statistician at the RAND Corporation in Santa Monica, California. Lisa Rubenstein is Professor Emeritus at UCLA Geffen School of Medicine and UCLA Fielding School of Public Health, and Physician Policy Researcher at RAND.

\section{Author disclosures}

The authors report no actual or potential conflicts of interest with regard to this article.

\section{Disclaimer}

The opinions expressed herein are those of the authors and do not necessarily reflect those of Federal Practitioner, Frontline Medical Communications Inc., the US Government, or any of its agencies.

\section{References}

1. Hayes SL, Salzberg CA, McCarthy D, et al. High-need, high-cost patients: who are they and how do they use health care? A population-based comparison of demographics, health care use, and expenditures. Issue Brief (Commonw Fund). 2016;26:1-14.

2. Bowman MA. The complexity of family medicine care. J Am Board Fam Med. 2011;24(1):4-5. doi:10.3122/jabfm.2011.01.100268

3. Grant RW, Adams AS, Bayliss EA, Heisler M. Establishing visit priorities for complex patients: a summary of the literature and conceptual model to guide innovative interventions. Healthc (Amst). 2013;1(3-4):117-122. doi:10.1016/j.hjdsi.2013.07.008

4. Okunogbe A, Meredith LS, Chang ET, Simon A, Stockdale SE, Rubenstein LV. Care coordination and provider stress in primary care management of highrisk patients. J Gen Intern Med. 2018;33(1):65-71. doi:10.1007/s11606-017-4186-8

5. Weiner JZ, McCloskey JK, Uratsu CS, Grant RW. Primary care physician stress driven by social and financial needs of complex patients. J Gen Intern Med. 2019;34(6):818819. doi:10.1007/s11606-018-4815-x

6. Shanafelt TD, Sloan JA, Habermann TM. The wellbeing of physicians. Am J Med. 2003;114(6):513-519. doi:10.1016/s0002-9343(03)00117-7

7. Loeb DF, Bayliss EA, Candrian C, deGruy FV, Binswanger IA. Primary care providers' experiences caring for complex patients in primary care: a qualitative study. $B M C$ Fam Pract. 2016;17:34. Published 2016 Mar 22. doi:10.1186/s12875-016-0433-z

8. Peek CJ, Baird MA, Coleman E. Primary care for patient complexity, not only disease. Fam Syst Health. 2009;27(4):287-302. doi:10.1037/a0018048

9. Powers BW, Chaguturu SK, Ferris TG. Optimizing highrisk care management. JAMA. 2015;313(8):795-796. doi:10.1001/jama.2014.18171

10. Skinner HG, Coffey R, Jones J, Heslin KC, Moy E. The effects of multiple chronic conditions on hospitalization costs and utilization for ambulatory care sensitive conditions in the United States: a nationally representative cross-sectional study. BMC Health Serv Res. 2016;16:77. Published 2016 Mar 1. doi:10.1186/s12913-016-1304-y

11. Zulman DM, Pal Chee C, Wagner TH, et al. Multimorbidity and healthcare utilisation among high-cost patients in the US Veterans Affairs Health Care System. BMJ Open. 2015;5(4):e007771. Published 2015 Apr 16. doi:10.1136/bmjopen-2015-007771

12. Breland JY, Asch SM, Slightam C, Wong A, Zulman DM. Key ingredients for implementing intensive outpatient programs within patient-centered medical homes: a literature review and qualitative analysis. Healthc (Amst). 2016;4(1):22-29. doi:10.1016/j.hjdsi.2015.12.005

13. Bodenheimer T. Coordinating care--a perilous journey through the health care system. $N$ Engl J Med. 2008;358(10):1064-1071. doi:10.1056/NEJMhpr0706165

14. Press MJ. Instant replay--a quarterback's view of care coordination. N Engl J Med. 2014;371(6):489-491. doi:10.1056/NEJMp1406033

15. Chang ET, Piegari RI, Zulman DM, et al. High-risk patients in VHA: where do they get their primary care? Abstract presented at the 2017 Society of General Internal Medicine Annual Meeting. J Gen Intern Med. 2017;32(suppl 2):83-808. doi:10.1007/s11606-017-4028-8

16. Chang ET, Zulman DM, Asch SM, et al. An operationspartnered evaluation of care redesign for high-risk patients in the Veterans Health Administration (VHA): Study protocol for the PACT Intensive Management (PIM) randomized quality improvement evaluation. Contemp Clin Trials. 2018;69:65-75. doi:10.1016/j.cct.2018.04.008

17. Olmos-Ochoa TT, Bharath P, Ganz DA, et al. Staff perspectives on primary care teams as de facto "hubs" for care coordination in VA: a qualitative study. J Gen Intern Med. 2019;34(suppl 1):82-89. doi:10.1007/s11606-019-04967-y

18. Iovan S, Lantz PM, Allan K, Abir M. Interventions to decrease use in prehospital and emergency care settings among super-utilizers in the United States: a systematic review. Med Care Res Rev. 2020;77(2):99-111. doi:10.1177/1077558719845722

19. Zulman DM, Ezeji-Okoye SC, Shaw JG, et al. Partnered research in healthcare delivery redesign for high-need, high-cost patients: development and feasibility of an Intensive Management Patient-Aligned Care Team (ImPACT). J Gen Intern Med. 2014;29 Suppl 4(Suppl 4):861869. doi:10.1007/s11606-014-3022-7

20. Chang ET, Raja PV, Stockdale SE, et al. What are the key elements for implementing intensive primary care? A multisite Veterans Health Administration case study. Healthc (Amst). 2018;6(4):231-237. doi:10.1016/j.hjdsi.2017.10.001

21. Rich E, Lipson D, Libersky J, Parchman M; Mathematica Policy Research. Coordinating care for adults with complex care needs in the patient-centered medical home: challenges and solutions. Published January 2012. Accessed January 12, 2021. https://pcmh.ahrq.gov/page /coordinating-care-adults-complex-care-needs-patient -centered-medical-home-challenges-and-0

22. Yoon J, Chang E, Rubenstein LV, et al. Impact of primary care intensive management on high-risk veterans' costs and utilization: a randomized quality improvement trial [published correction appears in Ann Intern Med. 2018 Oct 2;169(7):516]. Ann Intern Med. 2018;168(12):846-854. doi:10.7326/M17-3039 